\title{
Changes in Appetite During the Heart Failure Trajectory and Association With Fatigue, Depressive Symptoms, and Quality of Life
}

Christina Andreae, Martje H. L. van der Wal, Dirk J. van Veldhuisen, Bei Yang, Anna Strömberg and Tiny Jaarsma

The self-archived postprint version of this journal article is available at Linköping University Institutional Repository (DiVA):

http://urn.kb.se/resolve?urn=urn:nbn:se:liu:diva-171162

N.B.: When citing this work, cite the original publication.

Andreae, C., van der Wal, M. H. L., van Veldhuisen, D. J., Yang, B., Strömberg, A., Jaarsma, T., (2020), Changes in Appetite During the Heart Failure Trajectory and Association With Fatigue, Depressive Symptoms, and Quality of Life, Journal of Cardiovascular Nursing.

https://doi.org/10.1097/JCN.0000000000000756

Original publication available at:

https://doi.org/10.1097/JCN.0000000000000756

Copyright: Lippincott, Williams \& Wilkins

http://www.lww.com/ 


\begin{abstract}
Background: Decreased appetite can contribute to malnutrition in patients with heart failure. Little is known about the trajectory of appetite over time in patients with HF and the factors associated with decreased appetite after discharge from the hospital.
\end{abstract}

Objective: To investigate changes in appetite over time, and explore how fatigue, depressive symptoms and quality of life are associated with decreased appetite.

Methods: Data from the multicenter randomized Coordinating study evaluating Outcomes of Advising and Counselling in Heart failure $(\mathrm{COACH})$ were used. Logistic regression and mixed-effects logistic regression were used to investigate changes in appetite over time and to explore the relationship between appetite and fatigue, depressive symptoms, and quality of life.

Results: A total of 734 patients with HF (mean age 69 years) were included. Decreased appetite was present at all follow-up measurement, however, decreased appetite was significantly lower at 1 month OR 0.43 (CI 0.29-0.63), 6 months OR 0.31 (CI 0.20-0.47), 12 months OR $0.22(0.14-0.34)$ and 18 months' follow-up OR 0.24 (CI 0.15-0.37) compared to baseline. Decreased appetite was associated with fatigue OR 3.09 (CI 1.98-4.84), symptoms of depression OR 1.76 (CI 1.35-2.29) and low quality of life OR 1.01, (CI 1.01-1.02) across all measurement points adjusted for covariates.

Conclusions: Appetite improved post discharge, however at all time point at least $22 \%$ of patients reported decreased appetite. Fatigue, symptoms of depression and low quality of life are factors associated with decreased appetite. Decreased appetite is a long-standing problem in that does not disappear spontaneously after an acute HF deterioration.

Key words: Appetite, Depressive Symptoms, Fatigue, Heart Failure, Quality of Life 


\section{Introduction}

Decreased appetite, synonymous with anorexia, (1) can lead to malnutrition which in turn results to poor health outcomes such worsened functional capacity, morbidity and mortality (2-4). Decreased appetite in patients with heart failure (HF) may be driven by abnormal inflammatory processes, but also by disease-specific factors such as shortness of breath and gastrointestinal edema (5-7). It can also be caused by changes of hormones associated with older age, such as reduced feelings of hunger, altered satiety and diminished gastro motility $(1,8)$. In addition, depressive symptoms and fatigue, which are common in patients with HF (9-11), might play a role in causing a decreased appetite. Furthermore studies describe a relationship between psychological factors and a decreased appetite (12), although not performed in a HF population.

In research, the assessment of decreased appetite is mostly performed by determining appetite hormones in blood samples instead of the self-report of patient $(8,12)$. However, in clinical practice self-report of appetite, although not routinely performed, can be used to tailor treatment and self-care advice. Clinicians and patients themselves might expect that a decreased appetite will be resolved when patients are discharged from hospital to their normal environment and food routines, however, there is limited data on the change in appetite over time. A better understanding of decreased appetite might improve early detection of decreased appetite during the HF trajectory and help to design future interventions to reduce the risk or delay of the development of malnutrition. Therefore, the purpose of this study was to 1) investigate changes of appetite over time in patients with $\mathrm{HF}$ and 2) explore how fatigue, depressive symptoms and quality of life are associated with decreased appetite after a hospitalization for HF. 


\section{Methods}

\section{Design and sample}

Data from the multicenter randomized Coordinating study evaluating Outcomes of Advising and Counselling in Heart failure $(\mathrm{COACH})$ were used for secondary analysis. The $\mathrm{COACH}$ study was a randomized controlled trial that investigated the effect of nurse-led interventions on clinical outcomes in patients diagnosed with HF who were classified in New York Heart Association Classification (NYHA class) II-IV. Patients were randomized to control group "care as usual" or intervention group "basic support vs intensive support". The intervention of basic support consisted routine management by cardiologist and education by heart failure nurse according to guidelines, counselling based on individual education needs and follow up every 3 months. The intensive support consisted of similar program as basic support plus more intense follow up monthly with multidisciplinary support and 2 home visits. A total of 1023 patients were included during a hospitalization for HF. Data were collected at baseline, 1, 6, 12 and 18-months' follow-up. The study complied with the Declaration of Helsinki and was approved by ethical committee. All patients provided written consent to participate in the study. The methods and main results of the $\mathrm{COACH}$ study have been described in detail elsewhere $(13,14)$. In 1023 patients, $272(26 \%)$ patients died during the 18 month follow up. In this study, patients with completed appetite measures were included which resulted to 734 (71\%) patients at baseline.

\section{Measurements}

Decreased appetite was measured in an interview at baseline i.e., at discharge from hospital, and at 1, 6, 12 and 18 months with the question: 'Did you have a decreased appetite during the last month? (yes/no)'. In the current analysis the answer 'yes' was used to report 'decreased appetite'. Comparable single item appetite question has been used in other studies. Saitoh et al investigated decreased appetite in 166 clinically stable patients with HF on basis 
of a single item interview question "Do you have appetite loss" which was responded on a 6point Likert scale and dichotomized to $\geq 1$ as decreased appetite. In that study, decreased appetite predicted lower survival (2). In Huang et al study, 1856 community dwelling adults were interviewed with a single question to assess current appetite, responded to a three-stage response scale, good, faire, poor appetite. Those patients having poor appetite had nutrition deficiency of energy and certain minerals and vitamins compared to better appetite (15). The way of knowing patients appetite by using single item question has value in clinical settings and can be used to recognize patients with risk for limited food intake resulting to malnutrition (16).

Fatigue was measured in the same way with the question: 'Did you suffer from fatigue in the last month? (yes/no)'.

Depressive symptoms were measured with the self-reported Center for Epidemiologic Studies Depression Scale (CES-D). The instrument contains 20 items measuring depressive feelings and behaviors on a scale ranging from 0 (rarely or none of the time) to 3 (most or all of the time). The total score ranges from 0 to 60 , with higher scores indicating more depressive symptoms. CES-D has acceptable psychometrics sounds include concurrent and construct validity as well as acceptable test-retest (17). A cut-off point of 16, which is generally used to define patients at risk of clinical depressive symptoms was used to dichotomize patients into two groups; a score $\geq 16$ points indicate more symptomatology $(17,18)$.

The Minnesota Living with Heart Failure Questionnaire (MLHFQ) was used to measure quality of life during the past month. The instrument contains 21 items scored with a 6-point Likert type rating scale and has a response score 0 (none or not applicable), and from 1 (very little) to 5 (very much). The total score ranges from 0 to 105 , and a higher score indicates lower quality of life (19). MLHFQ has shown acceptable construct validity (20, 21). 
Demographic and clinical data on left ventricular ejection fraction (LVEF), NYHA-class, six minute walk test (6MWT), pharmacological treatment, blood samples of cardiac stress B-type natriuretic peptide (BNP), C-reactive protein (CRP) and anthropometrics were derived from medical records. Assessments were completed at discharge from hospital except CRP and BNP that were assessed at admission to hospital.

\section{Analysis}

Descriptive statistics including mean and standard deviation, median and interquartile range, frequency and percentage were used to describe demographic and clinical characteristics. Frequencies of patients who had a decreased appetite at baseline, 1 month, 6 months, 12 months and 18 months were calculated. The incomplete appetite measurements at any time at the follow-up were omitted.

Aim 1 was to explore changes in appetite over time. Logistic regression was performed where the outcome variable 'decreased appetite' was grouped to 'decreased appetite' versus 'no decreased appetite'. A p-value of $<0.05$ was used to evaluate the prediction of time at $1,6,12$, and 18 months of decreased appetite compared to baseline. The time variable at baseline was used as reference category. In addition, the study group assignment was treated as covariate in this study (13).

Aim 2 was to explore the associations between the explanatory variables fatigue, depressive symptoms, quality of life, and the outcome variable appetite which were measured at baseline, 1, 6, 12 and 18 months. Simple and mixed effects logistic analysis were performed for each explanatory variable (22).

First, we examined the simple association between each explanatory variable and decreased appetite using simple mixed-effects logistic regression model.

To further explore the associations between each explanatory variable and decreased 
appetite excluding the impact of potential confounding we performed multivariable mixedeffects logistic regression analysis where each explanatory variable was adjusted for covariates that possibly could affect appetite i.e., age, sex, NYHA class, 6MWT, BNP, CRP, Body Mass Index kg/m² (BMI), angiotensin-converting enzyme (ACE), angiotensin II receptor blockers (ARB), beta-blockers, diuretics and the study group assignment. The multiple mixed-effects logistic regression analysis was performed by condition on the additive effect of the covariates. A p-value of 0.15 was assigned for entered covariates and 0.20 for removed covariates, and models were constructed by using stepwise elimination.

In addition, to evaluate the overall effect of the associations between explanatory variables and decreased appetite from baseline to 18 months follow-up, we used time as a random variable for each separate model $(23,24)$. Combining the Akaike information criterion (AIC), the best models were selected (23). The model of best fit fitting was evaluated by F test for fixed effects, and random effect was also evaluated with a normal test. Analyses were performed using IBM SPSS Statistics version 22.

\section{Results}

\section{$\underline{\text { Sample }}$}

A total of 734 patients with HF (mean age $69 \pm 12,61 \%$ male) were included. Most of the patients had NYHA class II $(n=405,55 \%)$ and III $(n=304,41 \%)$. None of the patients had underweight regarding BMI (27 \pm 5 ) (Table 1). At baseline, 17 (2\%) out of 751 appetite measures were omitted from the analysis because of randomly missing. Out of 751 , similar randomly missing were omitted from the analysis at 1 month $(n=68,9 \%), 6$ month $(n=91$, $12 \%), 12$ month ( $\mathrm{n}=81,11 \%)$ and 18 month follow-up $(\mathrm{n}=105,14 \%)$. 


\section{Decreased appetite over time}

At baseline, 49\% ( $n=359)$ of patients reported having a decreased appetite while decreased appetite was less frequent at 18 months' follow-up, 22\% (n=141) (Figure 1).

Decreased appetite was most frequent at discharge from hospital but significantly lower $(\mathrm{p}<0.001)$ during the follow-up at all time points compared to baseline. At 1 month follow-up, patients had 0.43 (CI 0.29-0.63) lower risk of decreased appetite compared to baseline. The risk of having a decreased appetite continued to decrease at 6 months OR $0.31(0.20-0.47)$, at 12 months OR $0.22(0.14-0.34)$, and at 18 months OR 0.24 (CI $0.15-0.37$ ) when adjusted for COACH study assignment (Table 2).

\section{$\underline{\text { Relationship fatigue and decreased appetite }}$}

With data including all time points, there were a significant relationship between fatigue and decreased appetite in simple mixed-effects logistic regression OR 2.21 (CI 1.72-2.84). The association remained significant and increased to OR 3.09 (CI 1.98-4.84) when we adjusted for identified covariates i.e., sex, NYHA class, 6MWT, ARB, CRP and the study group assignment. The results showed that patients with fatigue were 3.09 times more likely to have decreased appetite compared to those with no fatigue. The associations between fatigue and decreased appetite were significant $\mathrm{p}<0.001$ at all time points (Table 3 ).

\section{$\underline{\text { Relationship decreased depressive symptoms and appetite }}$}

There was a significant relationship between depressive symptoms and decreased appetite in simple mixed-effects logistic regression OR 2.03 (1.74-2.36). The association remained significant and decreased to OR $1.76(1.35-2.29)$ when we adjusted for identified covariates i.e., sex, NYHA class, 6MWT, ARB, beta-blockers, CRP and the study group assignment. In the adjusted models, patients with depressive symptoms were 1.76 (CI 1.35-2.29) times more likely of being affected by decreased appetite compared with those with no depressive 
symptoms. The associations between depressive symptoms and decreased appetite were significant $\mathrm{p}<0.001 \mathrm{using}$ all the time points data (Table 3 ).

\section{$\underline{\text { Relationship decreased quality of life and appetite }}$}

There was also a significant relationship between quality of life and decreased appetite in simple mixed-effects logistic regression OR 1.01 (1.01-1.02). The association remained significant but did not change OR 1.01 (1.01-1.02) when we adjusted for identified covariates which were sex, NYHA class, 6MWT, CRP, BMI and the study group assignment. The results showed that patients with a lower quality of life were 1.01 (CI 1.01-1.02) times more likely of being affected by decreased appetite than those with higher quality of life. The associations between quality of life and decreased appetite were significant $p<0.001$ (Table 3).

\section{Discussion}

In the present study, decreased appetite was reported by almost half of the patients (49\%) at the time of discharge from hospital. Although decreased appetite became less prevalent over time, it was still a problem after 18 months follow-up, 141 patients $(22 \%)$ reporting a decreased appetite. This is in contrast to findings of a study by Pilgrim et al who reported that decreased appetite was consistently poor over time in a study among elderly patients (25). A possible explanation could be that approximately $50 \%$ in the study of Pilgrim had a hospital admission before the follow-up at six month. In our previous research we have described similar patterns of recovery from symptoms after discharge; for example, change in sleep problems or change in thirst over time $(26,27)$. These studies showed that although in some patients symptoms may recover spontaneously, in others they are persistent or even appear for the first time during the recovery process. In the current study we also described a persistent prevalence of a decreased appetite in patients, and this is important to consider. Health care 
providers might think that discharging patients to their own home and regular food habits will solve possible problems with appetite. However, our data show that not all patients spontaneously recover, and patients might be in need of more care related to their appetite and food intake during the whole disease trajectory. Multi-intervention studies designed to improve self-care after discharge from hospital are ongoing (28). Similar, supporting food habits to delay or prevent malnutrition in patients with poor appetite could be a target. For example, patients might not manage to prepare their daily meals because of the HF burden and might therefore need support with home delivered meals, assisted for example by home care services (29). Also, dietitian can provide supplements that can be helpful for patients in situations where HF symptoms, physical and mental status affect nutrition negatively. These supporting actions should however be based on accurate clinical nutrition assessment and individual's nutrition needs and preferences. Appetite should preferably be evaluated during the HF trajectory to prevent malnutrition (30).

None of the patients were underweight according to BMI, however BMI is primary used to define overweight and is not a good measure to identify nutrition status alone (31). To assess nutrition status, a number of assessments as food habits, dietary intake, laboratory tests, anthropometric measurements, clinical and etiological need to be judged (32).

In the present study, we found that fatigue was associated with a higher risk of developing decreased appetite. The relationship between fatigue and decreased appetite is complex and the direction of causation are not clear. Fatigue and decreased appetite are key components of malnutrition (16). Previously higher levels of fatigue and decreased appetite were found in patients with malnutrition compared with non-malnutrition (33). Both fatigue and decreased appetite need to be considered as important in preventing or delaying the development of malnutrition. 
In our study, we found that patients with depressive symptoms were more likely to be affected by decreased appetite compared with those without depressive symptoms. Our results are in line with findings from Landi et al which showed that patients with depressive symptoms had a two-fold higher risk for decreased appetite in a sample of elderly individuals (34).

Depressive symptoms need to be recognized as a potential risk factor for decreased appetite in older people $(35,36)$. Studies have also showed that depressive symptoms are associated with vitamin deficiency (37). Thus, depressive symptoms may be linked to both decreased appetite and vitamin deficiency that probably are close connected to each other.

In addition, we found that patients with impaired quality of life also had more often a decreased appetite than those with better quality of life. These results confirm studies in patients with cancer that described that decreased appetite is associated with quality of life (38). In a HF study, patients with better appetite reported better quality of life. However, these associations were only evident for those patients without depressive symptoms (39).

Decreased appetite is certainly a complex symptom which seems to go far beyond the pathophysiological perspective and therefore health care professionals should have a wider perspective on appetite, for example a sedentary lifestyle such number of steps per day was associated with appetite in patients with HF (40).

This study has some limitations. Decreased appetite was assessed with one question and two response alternatives which may limit the ability to understand appetite in compare if more appetite aspects are included. At present, there are no guidelines on how decreased appetite should be measured in patients with HF. To meet this limitation, we adjusted our analysis for potential factors that may influence appetite. Future research should preferably use validated instruments designed to assess appetite in patients with HF. 


\section{Conclusion and implications}

This study shows that decreased appetite is a prevalent problem in patients with HF, not only close to the time of hospitalization but also 1.5 years later. Moreover, fatigue, depressive symptoms and low quality of life were independently predictors of decreased appetite over time. Decreased appetite is a long-standing problem that does not disappear spontaneously, and fatigue, depressive symptoms and low quality contributes to the deterioration. Decreased appetite should be assessed in daily practice in the same way as other important nutritional assessments, such as weight. In that manner, nutritional advice could be given at an early stage to prevent or delay malnutrition. 


\section{References}

1. Landi F, Calvani R, Tosato M, Martone AM, Ortolani E, Savera G, et al. Anorexia of Aging: Risk Factors, Consequences, and Potential Treatments. Nutrients. 2016;8(2):69. 2. Saitoh M, Dos Santos MR, Emami A, Ishida J, Ebner N, Valentova M, et al. Anorexia, functional capacity, and clinical outcome in patients with chronic heart failure: results from the Studies Investigating Co-morbidities Aggravating Heart Failure (SICA-HF). ESC Heart Fail. 2017;4(4):448-57.

3. Sze S, Pellicori P, Kazmi S, Rigby A, Cleland JGF, Wong K, et al. Prevalence and Prognostic Significance of Malnutrition Using 3 Scoring Systems Among Outpatients With Heart Failure: A Comparison With Body Mass Index. JACC: Heart Failure. 2018;6(6):47686.

4. Bonilla-Palomas JL, Gamez-Lopez AL, Anguita-Sanchez MP, Castillo-Dominguez JC, Garcia-Fuertes D, Crespin-Crespin M, et al. Impact of malnutrition on long-term mortality in hospitalized patients with heart failure. Revista Espanola Cardiologia. 2011;64(9):752-8.

5. Anker SD, Sharma R. The syndrome of cardiac cachexia. International Journal of Cardiology 2002;85(1):51-66.

6. von Haehling S, Lainscak M, Springer J, Anker SD. Cardiac cachexia: a systematic overview. Pharmacol Ther. 2009;121(3):227-52.

7. Braun TP, Marks DL. Pathophysiology and treatment of inflammatory anorexia in chronic disease. J Cachexia Sarcopenia Muscle. 2010;1(2):135-45.

8. Malafarina V, Uriz-Otano F, Gil-Guerrero L, Iniesta R. The anorexia of ageing: physiopathology, prevalence, associated comorbidity and mortality. A systematic review. Maturitas. 2013;74(4):293-302. 
9. Johansson P, Lesman-Leegte I, Svensson E, Voors A, van Veldhuisen DJ, Jaarsma T.

Depressive symptoms and inflammation in patients hospitalized for heart failure. Am Heart J. 2011;161(6):1053-9.

10. Ghosh RK, Ball S, Prasad V, Gupta A. Depression in heart failure: Intricate relationship, pathophysiology and most updated evidence of interventions from recent clinical studies. Int $J$ Cardiol. 2016;224:170-7.

11. Williams BA. The clinical epidemiology of fatigue in newly diagnosed heart failure. $B M C$ Cardiovascular Disorders. 2017;17(1):122.

12. Ezeoke CC, Morley JE. Pathophysiology of anorexia in the cancer cachexia syndrome. $J$ Cachexia Sarcopenia Muscle. 2015;6(4):287-302.

13. Jaarsma T, Van Der Wal MH, Hogenhuis J, Lesman I, Luttik ML, Veeger NJ, et al. Design and methodology of the COACH study: a multicenter randomised Coordinating study evaluating Outcomes of Advising and Counselling in Heart failure. Eur J Heart Fail. $2004 ; 6(2): 227-33$.

14. Jaarsma T, van der Wal MHL, Lesman-Leegte I, Luttik M-L, Hogenhuis J, Veeger NJ, et al. Effect of Moderate or Intensive Disease Management Program on Outcome in Patients With Heart Failure: Coordinating Study Evaluating Outcomes of Advising and Counseling in Heart Failure (COACH). Archives of Internal Medicine. 2008;168(3):316-24.

15. Huang YC, Wahlqvist ML, Lee MS. Appetite predicts mortality in free-living older adults in association with dietary diversity. A NAHSIT cohort study. Appetite. 2014;83:89-96.

16. Evans WJ, Morley JE, Argiles J, Bales C, Baracos V, Guttridge D, et al. Cachexia: a new definition. Clin Nutr. 2008;27(6):793-9.

17. Radloff LS. The CES-D Scale:A Self-Report Depression Scale for Research in the General Population. Applied Psychological Measurement. 1977;1(3):385-401. 
18. Lesman-Leegte I, van Veldhuisen DJ, Hillege HL, Moser D, Sanderman R, Jaarsma T. Depressive symptoms and outcomes in patients with heart failure: data from the $\mathrm{COACH}$ study. Eur J Heart Fail. 2009;11(12):1202-7.

19. Rector TS. FDA Medical Device Development Tool (MDDT) Qualification Package for the Minnesota Living with Heart Failure Questionnaire (MLHFQ). 2017.

20. Heo S, Moser DK, Riegel B, Hall LA, Christman N. Testing the psychometric properties of the Minnesota Living with Heart Failure questionnaire. Nurs Res. 2005;54(4):265-72.

21. Bilbao A, Escobar A, Garcia-Perez L, Navarro G, Quiros R. The Minnesota living with heart failure questionnaire: comparison of different factor structures. Health Qual Life Outcomes. 2016;14:23.

22. Diggle PJ. Analysis of longitudinal data. New York: Oxford University Press; 2002.

23. Cnaan A, Laird NM, Slasor P. Using the general linear mixed model to analyse unbalanced repeated measures and longitudinal data. Statistics in Medicine. 1997;16(20):2349-80.

24. Dobson AJ, Barnett AG. An introduction to generalized linear models. Boca Raton: CRC Press; 2008.

25. Pilgrim AL, Baylis D, Jameson KA, Cooper C, Sayer AA, Robinson SM, et al. Measuring Appetite with the Simplified Nutritional Appetite Questionnaire Identifies Hospitalised Older People at Risk of Worse Health Outcomes. J Nutr Health Aging. 2016;20(1):3-7.

26. Johansson P, Brostrom A, Sanderman R, Jaarsma T. The Course of Sleep Problems in Patients With Heart Failure and Associations to Rehospitalizations. J Cardiovasc Nurs. 2015;30(5):403-10.

27. Waldreus N, van der Wal MH, Hahn RG, van Veldhuisen DJ, Jaarsma T. Thirst trajectory and factors associated with persistent thirst in patients with heart failure. J Card Fail. 2014;20(9):689-95. 
28. Jiang Y, Shorey S, Nguyen HD, Wu VX, Lee CY, Yang LF, et al. The development and pilot study of a nurse-led HOMe-based HEart failure self-Management Programme (the HOM-HEMP) for patients with chronic heart failure, following Medical Research Council guidelines. Eur J Cardiovasc Nurs. 2019:1474515119872853.

29. Buys DR, Campbell AD, Godfryd A, Flood K, Kitchin E, Kilgore ML, et al. Meals Enhancing Nutrition After Discharge: Findings from a Pilot Randomized Controlled Trial. Journal of the Academy of Nutrition and Dietetics. 2017;117(4):599-608.

30. Wilson MM, Thomas DR, Rubenstein LZ, Chibnall JT, Anderson S, Baxi A, et al. Appetite assessment: simple appetite questionnaire predicts weight loss in communitydwelling adults and nursing home residents. The American Journal of Clinical Nutrition. 2005;82(5):1074-81.

31. Jensen GL, Cederholm T, Correia M, Gonzalez MC, Fukushima R, Higashiguchi T, et al. GLIM Criteria for the Diagnosis of Malnutrition: A Consensus Report From the Global Clinical Nutrition Community. JPEN J Parenter Enteral Nutr. 2019;43(1):32-40.

32. Gibson RS. Principles of nutritional assessment. New York: Oxford University Press; 2005.

33. Zhou T, Yang K, Thapa S, Liu H, Wang B, Yu S. Differences in Symptom Burden Among Cancer Patients With Different Stages of Cachexia. J Pain Symptom Manage. 2017;53(5):919-26.

34. Landi F, Lattanzio F, Dell'Aquila G, Eusebi P, Gasperini B, Liperoti R, et al. Prevalence and potentially reversible factors associated with anorexia among older nursing home residents: results from the ULISSE project. Journal of the American Medical Directors Association. 2013;14(2):119-24.

35. de Boer A, Ter Horst GJ, Lorist MM. Physiological and psychosocial age-related changes associated with reduced food intake in older persons. Ageing Res Rev. 2013;12(1):316-28. 
36. Soenen S, Chapman IM. Body weight, anorexia, and undernutrition in older people. J Am Med Dir Assoc. 2013;14(9):642-8.

37. Song EK, Wu JR, Moser DK, Kang SM, Lennie TA. Vitamin D supplements reduce depressive symptoms and cardiac events in heart failure patients with moderate to severe depressive symptoms. Eur J Cardiovasc Nurs. 2018;17(3):207-16.

38. Barajas Galindo DE, Vidal-Casariego A, Calleja-Fernandez A, Hernandez-Moreno A, Pintor de la Maza B, Pedraza-Lorenzo M, et al. Appetite disorders in cancer patients: Impact on nutritional status and quality of life. Appetite. 2017;114:23-7.

39. Andreae C, Stromberg A, Chung ML, Hjelm C, Arestedt K. Depressive Symptoms Moderate the Association Between Appetite and Health Status in Patients With Heart Failure. J Cardiovasc Nurs. 2018;33(2):E15-E20.

40. Andreae C, Arestedt K, Evangelista L, Stromberg A. The relationship between physical activity and appetite in patients with heart failure: A prospective observational study. Eur $J$ Cardiovasc Nurs. 2019;18(5):410-417. 
What's New

- Half of patients with HF experienced decreased appetite during hospitalization of HF.

- Decreased appetite was most prevalent during the hospital stay and improved post discharge, but was still prevalent after discharge to 18 months later.

- Fatigue, symptoms of depression and quality of life are associated with decreased appetite and should be identified as possible factors associated with decreased appetite during the HF trajectory. 
Table 1. Demographic and clinical characteristics of patients with heart failure at baseline $(n=734)$.

\begin{tabular}{|c|c|}
\hline Age in years, mean (SD) & $69(12)$ \\
\hline Sex, male, n (\%) & $445(61)$ \\
\hline \multicolumn{2}{|l|}{ NYHA class, $\mathrm{n}(\%)$} \\
\hline II & $405(55)$ \\
\hline III & $304(41)$ \\
\hline IV & $18(2)$ \\
\hline Six minute walk test (meters), mean (SD) & $264(134)$ \\
\hline LVEF, mean (SD) & $34(14)$ \\
\hline BNP pg/mL, median (IQR) & $375(172-802)$ \\
\hline CRP (mg/l), mean (SD) & $4(6)$ \\
\hline BMI (kg/m2), mean (SD) & $27(5)$ \\
\hline \multicolumn{2}{|l|}{ Pharmacological treatment, n (\%) } \\
\hline Beta blocker & $501(68)$ \\
\hline ARB or ACE-inhibitor & $641(87)$ \\
\hline Diuretics & $701(95)$ \\
\hline Decreased appetite, n (\%) & $359(49)$ \\
\hline Fatigue, n $(\%)$ & $627(85)$ \\
\hline \multicolumn{2}{|l|}{ Depressive symptoms } \\
\hline Total score, mean (SD) & $15(11)$ \\
\hline Score $\geq 16, n(\%)$ & $266(36)$ \\
\hline Quality of life, mean (SD) & $43(22)$ \\
\hline
\end{tabular}


NYHA class, New York Heart Association (NYHA) functional classification; LVEF, Left Ventricular Ejection Fraction; BNP, B-type natriuretic peptide; CRP, C-reactive protein; BMI, Body mass Index. ARB, Angiotensin II Receptor Blocker; ACE inhibitors, Angiotensin-converting enzyme inhibitor. 


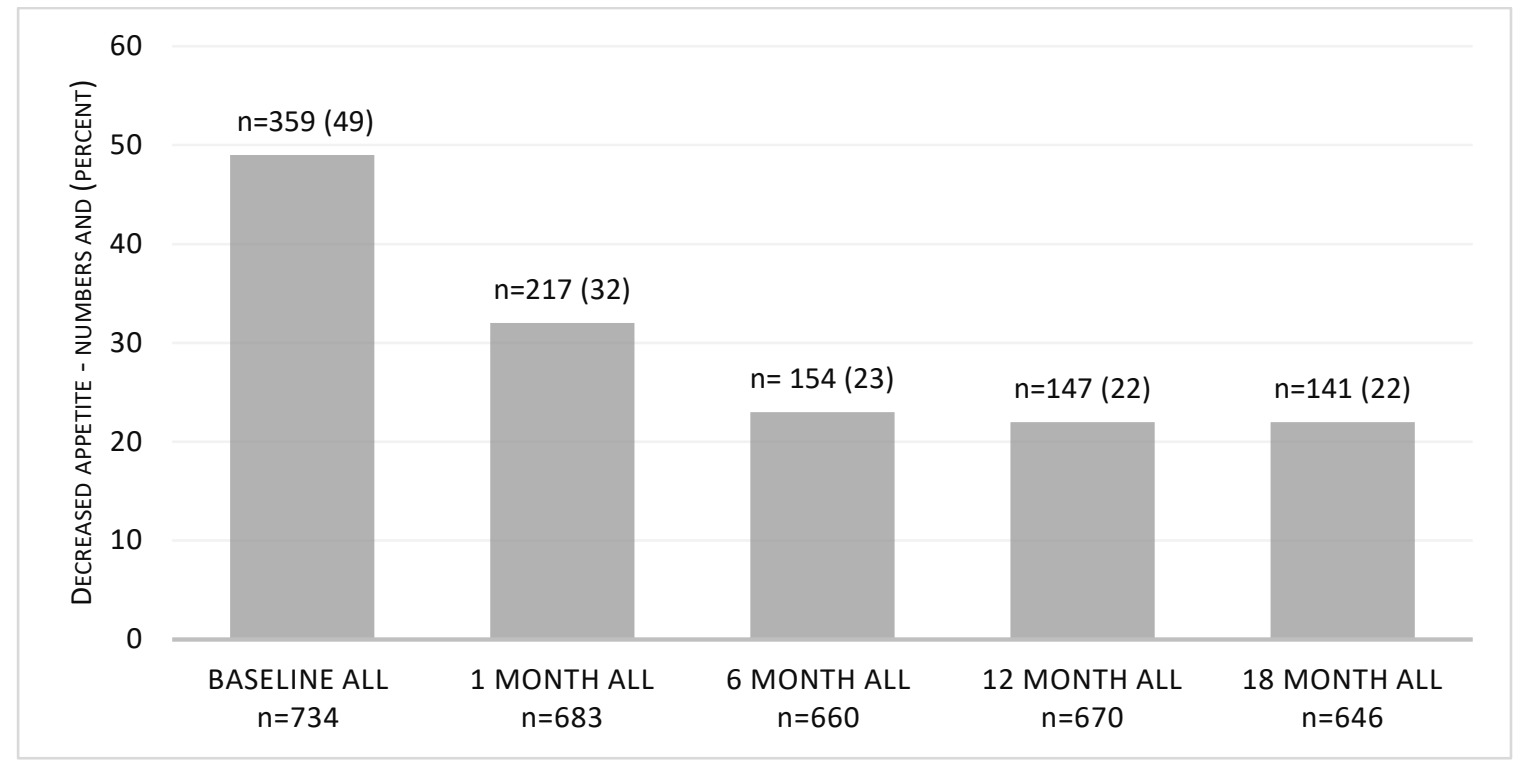

Figure 1. Prevalence n (\%) of decreased appetite of patients with heart failure at baseline, 1, 6, 12 and 18 months' follow-up. 
Table 2. Changes of decreased appetite of patients with heart failure over time from baseline at discharge from hospital to 18 months' follow-up based on logistic regression, $n=734$ adjusted for covariates.

Beta** $^{* *}$ S.E $\quad$ p-value $\quad$ Odds ratio and $95 \%$ confidence interval

Baseline (reference)

$\begin{array}{lcccc}1 \text { month } & -0.83 & 0.19 & <0.001 & 0.43(0.29-0.63) \\ 6 \text { month } & -1.16 & 0.21 & <0.001 & 0.31(0.20-0.47) \\ 12 \text { month } & -1.49 & 0.22 & <0.001 & 0.22(0.14-0.34) \\ 18 \text { month } & -1.40 & 0.22 & <0.001 & 0.24(0.15-0.37)\end{array}$

$\mathrm{R}^{2}=.071$ (Nagelkerke)

** $\log$ (Odds ratio)

Covariates: Study group assignment of the $\mathrm{COACH}$ study: care as usual or intervention basic support vs intensive support. 
Table 3. Associations between fatigue, depressive symptoms, quality of life of patients with heart failure at baseline and decreased appetite over time based on simple and multiple mixed effect logistic regression models adjusted for covariates.

\begin{tabular}{lllll}
\hline & Simple model & & $\underline{\text { Multiple model }}$ & \\
& OR $(95 \% \mathrm{CI})$ & p-value & OR (95\% CI) & p-value \\
\hline Fatigue $^{\mathrm{a}}$ & $2.21(1.72-2.84)$ & $<0.001$ & $3.09(1.98-4.84)$ & $<0.001$ \\
Depressive symptoms $^{\mathrm{b}}$ & $2.03(1.74-2.36)$ & $<0.001$ & $1.76(1.35-2.29)$ & $<0.001$ \\
Quality of life $^{\mathrm{c}}$ & $1.01(1.01-1.02)$ & $<0.001$ & $1.01(1.01-1.02)$ & $<0.001$
\end{tabular}

Fatigue $^{\mathrm{a}}=$ reference category no fatigue. Covariates included in the multiple model; sex, NYHA class, 6MWT, ARB,

CRP, study group assignment of the COACH study: care as usual or intervention basic support vs intensive support.

Depressive symptoms $^{\mathrm{b}}=$ reference category $<16$ points $=$ no depression. Covariates included in the multiple model; sex,

NYHA class, 6MWT, ARB, beta-blockers, CRP, study group assignment of the COACH study: care as usual or intervention basic support vs intensive support.

Quality of life ${ }^{c}=$ reference category is lower score which indicate less affected quality of life. Covariates included in the multiple model; sex, NYHA class, 6MWT, CRP, BMI, study group assignment of the COACH study: care as usual or intervention basic support vs intensive support. 